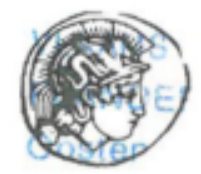

PERGAMON

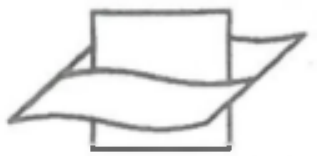

Vlaarns instituut voor de Zee

Fanders Marino Inatitute

Continental Shelf Research 18 (1998) 279-287
CONTINENTAL SHELF

RESEARCH

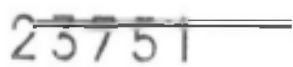

\title{
A two-compartment model for understanding the simulated three-dimensional circulation in Prince William Sound, Alaska
}

\author{
Eric Deleersnijder ${ }^{a, *}$, Jia Wang ${ }^{b}$, Christopher N.K. Mooers ${ }^{b}$

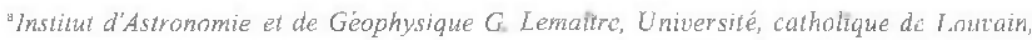 \\ 2 Chemin du Cyclotron, B-1348 Louvain-la-Neuve, Belgium \\ 'Ocean Prediction Fxperimental Laboratory and Division of Applicd Marine Physics, \\ Rosenstiel School of Marine and Atmospheric Science, University of Miami, 4600 Rickenbacker Causeway, \\ Miami, FL.33149-1098, L.S.A.
}

Received 15 July 1997: received in revised form 4 Augus1 1997; accepted 14 August 1997

\begin{abstract}
A two-compartment model of Prince William Sound (PWS), Alaska, is developed. One comparıment, corresponding to the southern PWS, represents advective phenomena, while the other is dominated by diffusion. This simple model is shown to reproduce rather well the temporal evolution of the mass of a passive tracer contained in PWS simulated hy a complex, three-dimensional model under five types of surface forcing. The three parameters of the box-model have clear physical meanings, which helps to understand the bydrodynamics of PW'S. In particular, the fraction of the flow entering the northern PWS is estimated, as wel] as the lurnover time of the two regions considered. (C) 1998 Elsevier Science Lid. All rights reserved
\end{abstract}

\section{Introduction}

One of the largest oil spills onto the seas occurred in Prince William Sound (PWS), Alaska, on 24 March 1989, as the result of a navigation error of the Exxon Valdez. Since then, considerable efforts have been devoted to the study of the ecosystem of this region. In this framework, Mooers and Wang (1997)- hereafter referred to as MW - used a version of the three-dimensional Princeton Ocean Model (e.g. Blumberg and Mellor, 1987) to simulate the water circulation in PWS.

${ }^{*}$ Corresponding author. Fax: + 32-10-474.722; e-mail: ericda astr.ucl.ac.be 
For details about the topography of the domain of interest, refer to MW. Herein, it is sufficient to mention that the lateral computational boundary is impermeable, except for two narrow passages in the southern part of PWS, Hinchinbrook Entrance and Montague Strait. Moreover, it must be underscored that, in general, water originating from the Gulf of Alaska enters PWS through the former and leaves through the latter.

MW conducted a sensitivity analysis of the PWS circulation to the surface stress. In the control run, no wind forcing was applied. Four additional simulations were carried out in which the wind was assumed to be blowing with the same speed toward the east, the north, the west and the south, respectively. For each type of forcing, a flow in statistical equilibrium was obtained. The latter was then used to simulate the fate of a passive tracer released at a constant rate for four days by a line source located in Hinchinbrook Entrance. This simulated tracer release will help to understand the circulation in PWS since a passive tracer parcel behaves like a water parcel.

In each model run, the source released tracer in the upper $40 \mathrm{~m}$ at the constant rate $\Phi$ from time $t=0$ until it was cut off, at time $t=T$, with $T=4$ days. Thus, the total amount of tracer injected into PWS was $M=\Phi T$. The tracer content of PWS, $m_{s}$, was simulated for 33 days by MW (Fig. 1).

The temporal evolution of the tracer mass present in PWS exhibits four distinct phases which are qualitatively similar in every model run (Fig. 2). First, from $t=0$ until $t=T, m_{s}$ grows approximately linearly, since almost no tracer parcel has yet left PWS through Montague Strait. Then, as long as the outgoing tracer flux is negligible, the tracer mass remains virtually constant. This regime lasts until $t=\tau$, which is the

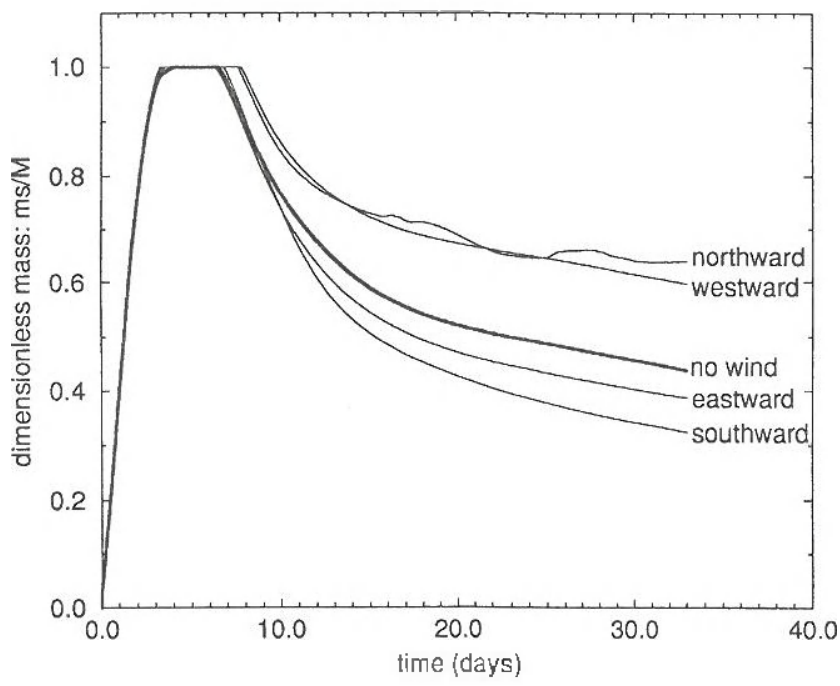

Fig. 1. The evolution of the dimensionless tracer mass present in PWS, $m$, $M$, as simulated by MW using the three-dimensional Princeton Ocean Model with various sulface forcings. The direction toward which the wind is blowing is indicated. (These curves are obtained by deleting the slight overshootings of the simulated mass, occurring for unidentified numerical reasons, after the source was cut off). 


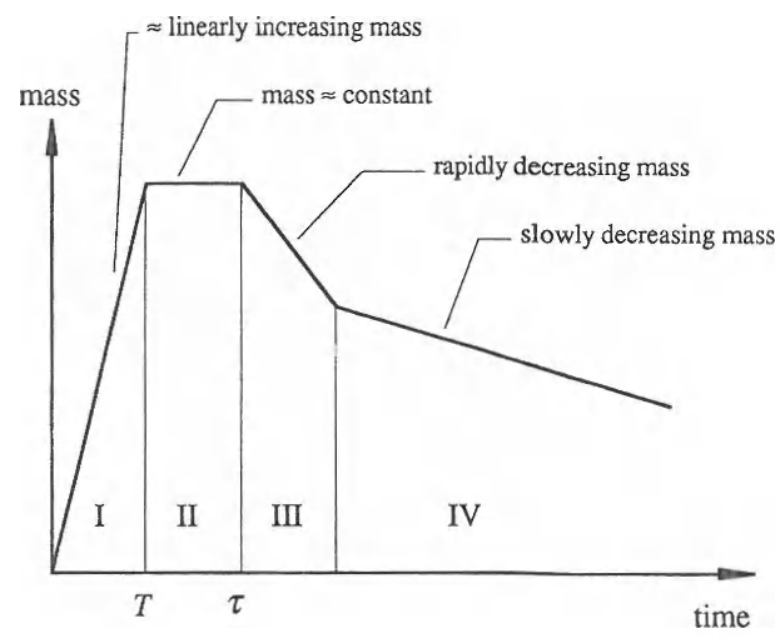

Fig. 2. Schematic illustration of the four phases, Iabelled I-IV, of the temporal evolulion of the tracer mass present in PWS.

time period that a tracer parcel needs to travel from Hinchinbrook Entrance to Montague Strait following the most direct pathway. Then, the tracer mass diminishes monotonically. During the third phase, the tracer mass decreases approximately Iinearly as time progresses. The transition from the third to the fourth phase is characterized by an abrupt change in the pace at which the mass diminishes. Finally, during the final phase, the rate of decrease of the mass is much smaller.

The processes which are at work during the first two phases are readily understood. That the mass must decrease during the subsequent phases is obvious. However; explaining why the rate of decrease of the mass changes is not straightforward. To do so, a simple, compartmental model is established, which will help infer major properties of the PWS circulation, as well as reveal the influence of the wind on them.

\section{A two-compartment model}

An attempt will be made to represent the evolution of PWS tracer content by means of an elementary model. The tracer mass estimated therefrom will be denoted $m$ so as to distinguish it from its counterpart, $m_{s}$, simulated by MW using a complex, three-dimensional model.

The tracer mass obeys

$$
\frac{\mathrm{d}}{\mathrm{d} t} m(t)=\phi^{\text {in }}(t)-\phi^{\text {out }}(t)
$$

where $\phi^{i n}$ is the tracer flux entering PWS through Hinchinbrook Entrance while $\phi^{\text {out }}$ represents the rate at which tracer leaves PWS through Montague Strait. The flux 
$\phi^{\text {in }}$ is due to the tracer source, while the outgoing flux depends on the tracer content of the Sound and the hydrodynamic processes occurring in it, the major features of which must be understood for a suitable parameterization to be devised.

The MW modelled current maps clearly suggest that a tracer parcel may follow two distinct types of path leading from Hinchinbrook Entrance to Montague Strait. The largest current speeds occur between Knight Island and Montague Island, implying that the quickest pathway is associated with advection through this region. The second route is that of parcels going further north into the Sound, where the circulation is much slower and intricate, partly because of numerous topographic features, such as islands, fjords, or headlands. At the scale of the northern PWS, these advective processes may amount to diffusion.

This description provides a basis for dividing PWS into two parts, the southern PWS - also termed 'region 1' below - dominated by advection and the northern PWS - also called 'region 2' hereafter - where diffusive mechanisms prevail. Therefore, the tracer mass present in PWS at a given time, $m(t)$, may be regarded as the sum of $m_{1}(t)$ and $m_{2}(t)$, where $m_{i}(t)(i=1,2)$ is the tracer mass contained in region $i$. If $\phi_{i}^{\text {in }}$ and $\phi_{i}^{\text {out }}$ denote the fluxes entering and leaving region $j$, respectively, $m_{i}$ obeys

$$
\frac{\mathrm{d}}{\mathrm{d} t} m_{i}(t)=\phi_{i}^{\text {in }}(t)-\phi_{i}^{\text {out }}(t), \quad i=1,2
$$

Since region 1 is assumed to be dominated by advection,

$$
\phi_{1}^{\text {out }}(t)=\phi_{1}^{i n}(t-\tau)
$$

where the time lag $\tau$, as already mentioned in the preceding section, is the period of time that a tracer parcel, following the quickest path, needs to transit from Hinchinbrook Entrance to Montague Strait.

If the diffusive processes taking place in region 2 are rather strong, then the tracer concentration therein may be assumed to be sufficiently homogeneous for the tracer flux leaving this region to be proportional to the tracer mass present in it, i.e.

$$
\phi_{2}^{\text {out }}(t)=\frac{m_{2}(t)}{\theta}
$$

where the time scale $\theta$ is the turnover time of region 2. The latter is defined to be the average over the northern PWS of the residence time - which, at a given point of region 2, is the period of time that a water parcel, initially located at the point considered, needs to leave the northern PWS. Additional explanations and appropriate references on such hydrodynamic time scales, as well as parameterizations similar to Eq. (4), may be found in Bolin and Rodhe (1973) and Tartinville et al. (1997).

Let $\alpha$ denote the fraction of the tracer flux entering PWS that goes directly into region (2). Hence,

$$
\phi_{2}^{i n}(t)=\alpha \phi^{i \prime \prime}(t)
$$

The tracer flux leaving region 2 may be assumed to join that exiting the southern PWS. However, it is readily seen that such a flux arrangement would prevent the PWS 


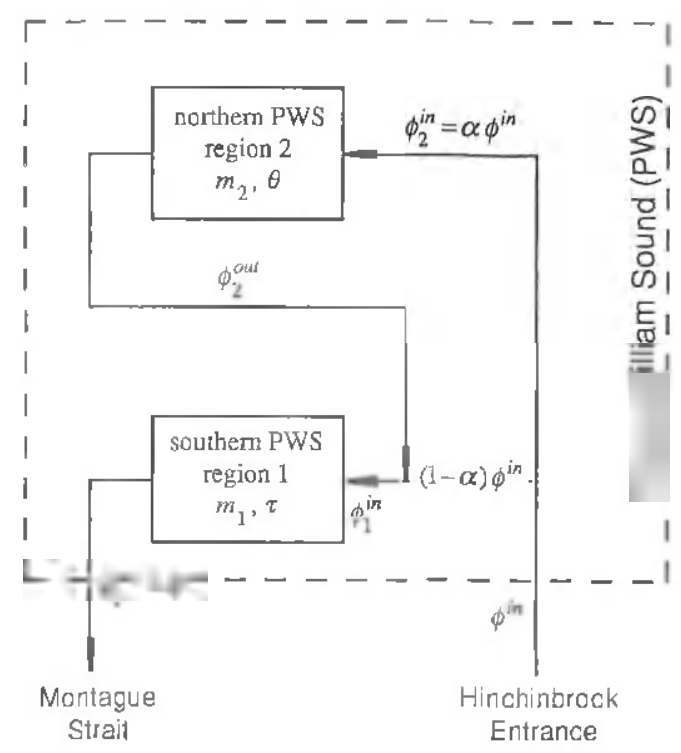

Fig 3. Schematic illustration of the tracer fluxes accounted for in the compartmental model.

tracer content from growing linearly during the first phase of the tracer mass evolution. In addition, $m$ could not remain almost constant for a certain period of time after the source is cut off. To circumvent these problems, the tracer pathway involving region 2 must include an appropriate time lag. For the sake of simplicity, it is decided that the flux leaving region 2 enters the southern PWS (Fig. 3), i.e.

$$
\phi_{1}^{\text {in }}(t)=(1-\alpha) \phi^{\text {in }}(t)+\phi_{2}^{\text {out }}(t)
$$

This is a modelling choice which does not require introducing a time lag specific to the northern PWS, since use is made of that associated with region 1.

The two-compartment model meant to represent the major features of the tracer evolution in PWS consists of Egs. (1)-(6). the solution of which reads

$$
m(t)=\int_{0}^{t}\left[\phi^{i n}(\mu)-\left(1-\alpha \mathrm{e}^{(\mu-t) / \theta}\right) \phi^{i n}(\mu-\tau)\right] \mathrm{d} \mu
$$

if $m(0)=0$. In the case considered above, $\phi^{i n}=\Phi$ if $0 \leqslant t \leqslant T(=4$ days $)$, and $\phi^{i n}=0$ otherwise. Therefore, if $T<\tau$,

$$
\begin{aligned}
& m(t)= \begin{cases}\Phi t, & 0 \leqslant t \leqslant T \\
\Phi T, & T \leqslant t \leqslant \tau\end{cases} \\
& m(t)=\Phi(T+\tau-t)+\alpha \Phi \theta\left(1-\mathrm{e}^{(\tau-\tau) / \theta}\right), \quad \tau \leqslant t \leqslant T+\tau
\end{aligned}
$$

and

$$
m(t)=\alpha \Phi \theta\left(\mathrm{e}^{T, \theta}-1\right) \mathrm{e}^{(\tau-t) / \theta}, \quad T+\tau \leqslant t
$$




\section{Assessment and discussion}

As expected, the solution above exhibits four different phases. It remains to be seen whether or not the three parameters $\tau, \theta$ an- $1 \mathrm{~d} \alpha$ may be calibrated in such a way that the evolution of the tracer content predicted by the compartmental model, $m$, is close to that simulated by $\mathrm{MW}, m_{s}$

The dimensionless distance between $m$ and $m_{s}$ may be defined as

$$
\varepsilon=\frac{\left[\int_{0}^{t_{\max }}\left(m-m_{s}\right)^{2} \mathrm{~d} t\right]^{1 / 2}}{\Phi T\left(t_{\max }\right)^{1 / 2}}
$$

where $t_{\max }=33$ days is the duration of MW's three-dimensional simulations. Obviously, the values of the parameters $\tau, \theta$, and $\alpha$ to be considered are those that minimize $\varepsilon$. They are obtained by means of a simple, iterative algorithm. A simple inspection of the three-dimensional model results (Fig. 1) suggests selecting 7 days, 50 days and 0.7 as the initial values of $\tau, \theta$ and $\alpha$, respectively. In fact, $\tau$ is the time elapsed at the end of the second phase of the evolution of the PWS tracer content. Next, $\alpha$ may be estimated from the slope of the tracer mass during phase III — as is illustrated by the asymptotic expansion (10) below. Finally, knowing $\tau, \theta$ may be approached through calculation of the ratio of the tracer mass at the beginning of the fourth phase to that at the end of the three-dimensional simulation period.

As may be seen in Table 1, for every type of surface forcing, $\varepsilon$ is rather small, indicating that there is an excellent agreement between the evolution of the tracer mass simulated by MW and that derived from the present compartmental model, which is clearly confirmed by the similarity of Figs. 1 and 4 .

The parameters of the simple model are such that $\tau-t \ll \theta$ during the third phase of the tracer mass evolution, i.e. during the time interval $\tau \leqslant t \leqslant T+\tau$. As a result, $m$ admits the following asymptotic expansion

$$
m(t) \approx \Phi[T-(1-\alpha)(t-\tau)], \quad \tau \leqslant t \leqslant T+\tau
$$

which is the reason why the tracer mass decreases almost linearly during the third phase, as may be seen in Figs. 1 and 4.

Table 1

The values of the parameters $\tau, \theta$ and $\alpha$ minimizing $\varepsilon$ as determined for various surface forcings. The direction toward which the wind blows is indicated. The error bars related to these estimates are $(\Delta \tau, \Delta \theta, \Delta \alpha)=(0.1$ day, 1 day, 0.01$)$

\begin{tabular}{lccccc}
\hline & Nowind & Westward & Southward & Fastward & Northward \\
\hline$\tau$ (days) & 7.1 & 7.8 & 7.4 & 7.1 & 7.5 \\
0 (days) & 48 & 80 & 32 & 47 & 97 \\
$\alpha$ & 0.68 & 0.78 & 0.62 & 0.62 & 0.78 \\
$\varepsilon$ & 0.065 & 0.066 & 0.067 & 0.066 & 0.066 \\
\hline
\end{tabular}




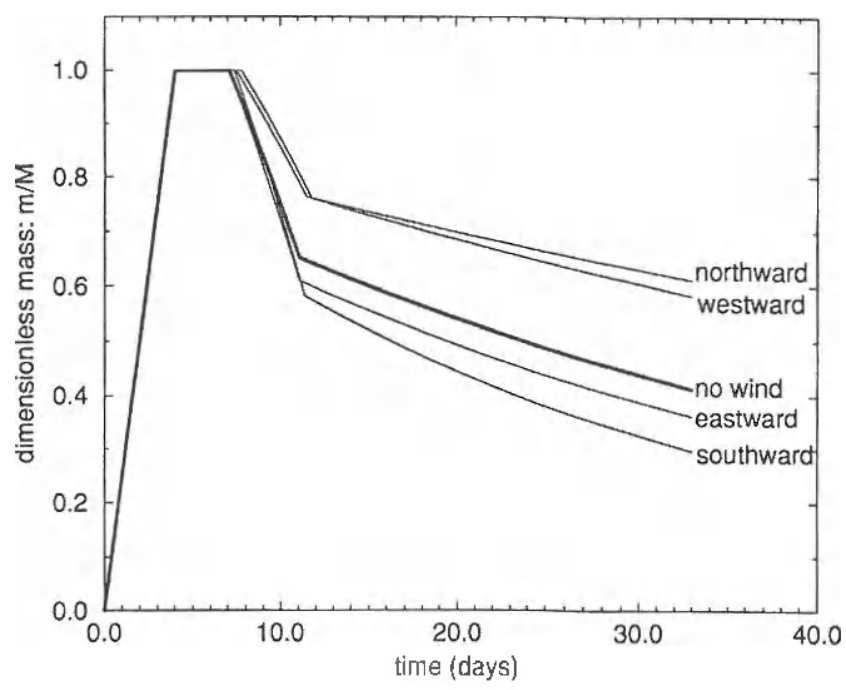

Fig. 4. The evolution of the dimensionless tracer mass present in PWS, $m / M$, obtained from the compartmental model with varjous surface forcings. The direction towards which the wind is blowing is indicated.

It is clear that the turnover time of the southern PWS, as modelled above, is equal to $\tau / 2$. That, in all cases, the turnover time of region 1 is much smaller than that of region 2 enables suggesting the following scenario: during the third phase, the outgoing flux at Montague Strait is essentially due to the tracer parcels that followed the quickest route, i.e. those that did not enter region 2; most of the tracer parcels which penetrated into the northern PWS leave PWS during the final phase.

As may be expected, the fraction of the tracer parcels entering the northen PWS, $\alpha$, is largest, or smallest, when the wind is northward, or southward, respectively. When the wind is northward or westward - which induces a net northward Ekman transport - it is conceivable that tracer parcels leave the northen PWS less frequently to enter the southern region, so that it is in such circumstances that the turnover time of region $2, \theta$, is largest (Table 1). Conversely, $\theta$ is smallest when the wind is southward or eastward.

For reasons which are still unclear, the advective time lag $\tau$ depends weakly on the surface forcing considered (Table 1). However, it may be that $\tau$ is dominated by the specified constant throughflow and not much influenced by the Ekman flow which varies from case-to-case, a hypothesis consistent with MW's modelled current fields.

It cannot be claimed that the simple model above is more realistic than the threedimensional Princeton Ocean Model applied by MW to PWS. That the compartmental model provides at almost no cost an estimate of the tracer transport through PWS is marginally interesting. since most three-dimensional models may now be run 
routinely in domains like PWS. The most appealing feature of the two-compartment model is that it involves only three parameters, which all have a clear physical meaning, helping to understand the hydrodynamics of PWS - since the tracer and water parcels behave similarly. So, it has been possible to estimate, for every type of surface forcing considered, the fraction of the water flux crossing Hinchinbrook Entrance which first goes northward, instead of flowing directly toward Montague Strait. In addition, the turnover times of the southern and the northern regions have been evaluated. Finally, it has been suggested that the hydrodynamic processes taking place in the latter region amount to diffusion, while the former is dominated by mere advective mechanisms.

To design the two-compartment model, it has nol been necessary to precisely delineate the boundary between the two regions considered. In fact, the only clear-cut difference between the two boxes is that they are dominated by hydrodynamic processes of a different nature, which does not prevent them from overlapping geographically. In other words, it is hydrodynamics rather than topography that allows distinguishing the two compartments. Thereforc, it would be difficult to validate the simple model by direct comparison with fluxes computed, or measured in situ, through a given section of PWS. This consideration is why an inverse approach has been - and should continue to be - preferred.

In spite of the excellent agreement between the evolution of the tracer mass simulated by MW's complex three-dimensional model and that predicted by the present simple model, the latter may not be deemed to be fully validated. In fact, it would be necessary to compare the predictions of the compartmental model to those of the three-dimensional model over periods of time larger than the largest turnover time, i.e. at least 100 days. On the other hand, for every type of flow, only one tracer release scenario has been considered so far, which is not sufficient. Therefore, for a given flow in PWS, it should be verified that the same set of parameters enables the two-box model to represent the evolution of the tracer mass in a satisfactory way throughout a series of tracer releases. For instance, a constant source located at Hinchinbrook Entrance of magnitude $\Phi$ may be considered, in which case the tracer mass should tend to $(\tau+\alpha \theta) \Phi$. Another interesting example consists of a source releasing an amount $M$ of tracer in an arbitrarily short period of time, which corresponds to the case detailed above in the limit $T \rightarrow 0$. In this case, according to the simple model, the tracer mass should remain equal to $M$ until $t=\tau$; then $m$ should undergo a discontinuity, and finally decrease as $\alpha \exp [(\tau-t) ; 0]$ for $\tau<t$.

\section{Acknowledgements}

ED is a Research Associate with the National Fund for Scientific Research of Belgium. JW and CNKM appreciate the financial support of the Sound Ecosystem Assessment (SEA) Program of the Exxon Valdez Oil Spill (EVOS) Trustees Council through Prince William Sound Science Center, Alaska. ED is indebted to Benoit Tartinville and Marian Scott for the comments they provided on various aspects of this work. 


\section{References}

Blumberg, A.F.. Mellor. G.L., 1987. A description of a three-dimensional cnastal ocean circuiaticn model In: Heaps, N.S., (Ed.), Three-Dimensional Coastal Ocean Modeis. American Geophysical Union, Washington, DC, pp. $1-16$.

Bolin, B.. Rodhe, H., 1973. A note on the concepts of age distribution and transit time in natural resersoirs. Tellus 25, 58-62.

Mooers, C.N.K., Wang. J., 1997. On the implementation of a three-dimensional circulation model for Prince William Sound, Alaska, Continental Shclr Research, 18, 253-277.

Tartinville, B.. Deleersnijder. E.. Rancher. J., 1997. The water residence time in the Mururos atoll Iagoon: sensitivity analysis of a three-dimensional model. Coral Reefs, 16. 193-203. 
